\title{
Herd Behavior in Financial Markets
}

\author{
Wenjin Kang ${ }^{1}$ \\ ${ }^{1}$ National University of Singapore, Singapore \\ Correspondence: Wenjin Kang, Department of Finance, NUS Business School, National University of \\ Singapore, Singapore 119245, Singapore. Tel: 65-6516-3194. E-mail: bizkwj@nus.edu.sg
}

Received: February 3, 2013

Accepted: April 19, $2013 \quad$ Online Published: May 17, 2013

doi:10.5539/ibr.v6n6p31

URL: http://dx.doi.org/10.5539/ibr.v6n6p31

\begin{abstract}
This paper provides a rational microstructure model in which informed traders have private information on multi-dimensional uncertainties and it is possible for the herd behavior to occur. In the herd phase, informed traders choose to trade in the same direction regardless of their private signals. In addition, we find that there always exists a transitional phase between the normal phase and the herd phase. The market enters a transition phase when some informed traders' expectation on the risky asset value is within the bid-ask spread. In the transition phase, the market liquidity deteriorates, marked by larger spread and stronger price impact power of incoming orders. The research in the multiple markets situation shows that what happens in the transition phase in one market could affect not only that market but also related markets. It provides the stage for the market crash and the herd behavior to be contagious among multiple assets whose values are correlated.
\end{abstract}

Keywords: herd behavior, multi-dimensional information, liquidity

\section{Introduction}

The history of financial markets is punctuated with menacing market crashes. In order to explain market crash mechanisms, the theory of herd behavior and informational cascade have been widely exploited by scholars. The herding literature originates from Bikhchandani, Hirshleifer, and Welch (1992, BHW hereafter). BHW show that individuals can rapidly converge on one action based on little information, and an informational cascade arises when the individual's action does not depend on his private signal and the information flowing into the market is blocked. Banerjee (1992) construct a sequential decision model in which the optimizing individuals' decision rules can be characterized as herd behavior, that is, people will do what others are doing rather than using their own information.

In the related empirical literature, Grinblatt, Titman, and Wermers (1995) find some evidence that mutual funds tend to buy and sell the same stocks at the same time. Trueman (1996) shows that there is a tendency for analysts to release forecasts closer to those previously announced by other analysis, even when this is not justified by their information. Lee (1998) finds that, in a financial market with sequential trading structure, the transaction cost may prevent informed traders to reveal their private information and trade in a sequential fashion. Nofsinger and Sias (1999) show that institutional investors exhibit herding behavior pattern, which is positively correlated with lag returns and also is related to stock return momentum. More recently, Ouarda, Bouri, and Bernard (2013) find strong evidence of herding behavior in European stock markets, which can lead to bearish market situation characterized by higher volatility and volume. Lao and Singh (2013) show that herding behavior exists in both Chinese and Indian stock markets.

One important feature of the BHW (1992) model is that the adoption cost, which can be interpreted as the market price in a financial market, is fixed. In BHW (1992) basic model, the asset payoff is 0 or 1 and the adoption cost is always $1 / 2$. If the individual observes his predecessors adopt and his expected adoption value is higher than $1 / 2$ even with a negative signal, he will simply follow his predecessors and adopt. Thus, this individual in fact chooses to herd and informational cascade occurs. However, in a financial market, the market maker will adjust his quotes dynamically according to the order flow. It is straightforward to show that, with a single-dimension signaling mechanism, the market price will always be adjusted so precisely that traders will trade in accord with their own private signals, and neither herd behavior nor informational cascades could happen, as described in Avery and Zemsky (1998). In order to make the herd behavior possible in a financial market with dynamic price adjustment, Avery and Zemsky (1998) assume multi-dimensional uncertainties in their model. They show that 
with the coexistence of value uncertainty and event uncertainty herd behavior is possible to happen. With an additional compositional uncertainty, a bubble and the burst can be simulated.

Inspired by their delicate information structure, we assume different kinds of uncertainty and information structure and extend the discussion into different directions in this paper. For example, few papers in the literature have discussed what will happen after a crash. As we know, after a crash, the market could behave very differently. Sometimes a fast and strong rebound follows the crash. For example, after the event in September of 2001, the S\&P 500 index took only one month to rebound to the level attained before the attack. On the other hand, the market may stay depressed in the aftermath of the crash for a much longer period. After the 1997 Asian crisis, it took the Hong Kong Hang Shen Index more than two years to climb back to the level it had attained before the crisis. A natural question is what causes these differences in recovery speed.

In this paper, we present a model with multiple dimensions of uncertainty to explain not only the crash itself but also how the market will behave after the crash. In particular, we assume that the asset value consists of two components, indicating a two-dimensional uncertainty structure. Each component has two possible states -- a high state and a low state. Only when both components are in the high state, can the final asset payoff be high. If either of these two components is in the low state, the asset payoff will be low. The informed trader receives two signals on these two components, separately. One signal is perfect, that is, if the first value component is in the high (low) state, the corresponding signal will be positive (negative). The other signal is noisy. If the second value component is in the high (low) state, this noisy signal will be positive (negative) with a probability larger than $1 / 2$ but less than 1 . Given the value structure and signal mechanism, if we assume the market is not in the herd phase, the informed trader will buy only when he receives a positive perfect signal and a positive noisy signal, and will sell when he receives either a negative perfect signal or a negative noisy signal combined with a positive perfect signal. A negative perfect signal received by the informed trader implies that the asset payoff is surely low. Hence we call it as the strong-negative signal. If the informed trader receives a negative noisy signal combined with a positive perfect signal, it indicates that the asset payoff is likely to be low, but not necessarily. We name it as the weak-negative signal. We also name the combination of a positive perfect signal and a positive noisy signal as the positive signal.

Thus, a market sell order has two possible explanations. It could be caused either by a strong-negative signal or a weak-negative signal. For the informed trader, these two types of signals are exclusive to each other. The informed trader can tell the difference, but the market maker cannot. He has two layers of uncertainty. Facing the income sell order, the market maker will place more weights on both the possibility of a strong-negative signal and the possibility of a weak-negative signal. Enhancing the latter belief will produce larger downward price pressure than the former since the asset value is definitely low with a negative perfect signal.

If there are consecutive and excessive sell orders on the market, the market maker will believe that it is likely to be caused by the informed trader receiving a strong-negative signal and will adjust his quotes downward aggressively sooner or later. Thus, a market crash can happen. If it is true that the perfect-signaled component is in the low state, the price will remain stable at the low level because every informed trader receives a strong-negative signal and knows that the final asset payoff is low. No rebound will happen after the crash in this scenario.

The model becomes more interesting when the realization of the perfect-signaled component is high. The informed trader knows this and will receive either a weak-negative signal or a positive signal. He only needs to infer the value of the noisy-signaled component from his own private noisy signal and the historical order flow in order to assess the asset payoff. The market maker, however, has to infer the value of not only the noisy-signaled component, but also the perfect-signaled component. Therefore, the herd behavior is possible because of the multiple-dimension uncertainty and the information asymmetry. Excessive sells can still lead to a market crash. If the market maker puts too much weight on the belief that of the perfect-signaled component is in the low state, which is not the truth here, and set the price too low, the informed trader's expectation of the asset value could be higher than the market price even if he receives a weak-negative signal. Thus, a herd buy, which is defined as every informed trader will buy regardless of his own private signal, could happen.

In addition, we find a particularly interesting transition phase existing between the normal phase and the herd phase. During the transition phase, some informed trader's expectation falls into the bid-ask spread and he will choose neither to buy nor to sell. In this transition phase, the market is more sensitive, and the bid-ask spread becomes larger than usual. Also, sell orders submitted in the transition phase has stronger price impact power and can tumble the price down quickly. It may lead to a market crash. If the price is set too low and there is a herd buy after the transition phase, it indicates a rebound following the crash. 
Next, we consider the situation of multiple assets sharing a common value component as the link of their value and address whether the trading in one market can trigger the herd behavior in another related market. We provide an interesting example in which it not only triggers but also significantly accelerates the occurrence of herd behavior in another market, because what happens in one market during its transition phase will substantially affect other related markets.

This paper is organized as follows: Section 2 introduces the economy and our assumptions for the model. In Section 3 we discuss the mechanism of herd behavior and the attributes of the transition phase before the herding. In Section 4, we move to a multiple-asset scenario and show an example of contagion of herd behavior. Finally, Section 5 concludes this paper and discusses some topics for future research.

\section{The Economy}

We first consider a market for one risky asset and one riskless asset. For the sake of simplicity, the time discount rate is zero, and the price of the riskless asset is used as the denominator. We assume that the risky asset pays no dividend and an infinite sequence of traders can arrive before its liquidation. The ex post liquidation value $Y$ of the risky asset is can be written as $Y=V W . W$ can be interpreted as the quality of the production technology of the firm, and $V$ is its management strength. There are two states of firm's technology, promising technology $\left(W_{H}\right)$ and fruitless technology $\left(W_{L}\right): W_{H}=1$ and $W_{L}=0$. Similarly, there are two states for $V$, that is, strong management $V_{H}=1$ and weak management $V_{L}=0$. The informed traders know whether the production technology is promising or not since it can be revealed by scientific research. They receive only noisy signals, however, about its management strength, since it cannot be easily quantified. When there are two companies using the same production technology, the technology itself is the same, but their management strength could be different. Hence, we could have two assets with $Y_{1}=V_{1} W$ and $Y_{2}=V_{2} W$, whose interaction will be discussed in Section 4 .

We can rewrite our information structure as $Y=1$ \{private signal is clear $(w=0)\} \times 0+1$ private signal is noisy $(w=1, v$ is noisy $)\} \times V$. This information structure represents a world of two possible natures: one is a world of no uncertainty to the informed traders, since their signal is always right; the other is a world where the informed traders' signal is noisy. $V$ is the informed trader's expectation of $Y$ according to his own noisy signal in the second nature. The informed traders know which nature the world belongs to, but the market maker does not. The nature $W=0$ implies that the profitability of the technology is so poor that no firm can earn a high return; the other nature $W=1, Y=V$ implies that the technology is good, but the individual firm needs to distinguish itself from its competitors by capable management to succeed. As we have said above, the informed traders know which nature the world belongs to, but the market maker doesn't.

A virtue of our model is that our information structure can be packed into a concise mathematical form, that is, $Y=V W$, where $W$ can serve as the common value linkage. For example, if we interpret $W$ as technology strength, it can serve as the link of asset values of different firms within the same industry that adopt the same production technology. Hence, our model is able to provide an explanation of how a crash could happen and be contagious among a group of assets whose values are related with each other. The motivation to design a common link of returns is that during a crash investors' concerns are usually not limited to a few individual firms. For example, in the burst of NASDAQ bubble in 2000, high-technology stocks were hit most, and then in the recent financial crisis in 2008, it is financial industry that investors worry more.

In our model, risk-neutral traders arrive on the market sequentially, indexed by the time $t=1,2,3, \ldots \ldots$. They have the option to buy or sell one unit of stock or choose not to trade. This model actually allows a trader to trade more than one unit implicitly. If the trader wants to trade $n$ units, we can treat the trader as presenting trade orders at time $\{t, t+1, \ldots, t+n-1\}$ with one unit of trade per time. The average of these $n$ trading prices is equal to the actual price the trader pays. Two types of traders exist: the informed and uninformed trader. The possibility of a trader being informed at any given time is $\mu$, and the possibility of being uninformed is $1-\mu$. Informed traders know $W$ precisely; i.e., their signal $w$ about $W$ is always right, and they receive a noisy private signal $v_{i}$ regarding the true value of $V$, with $\operatorname{Pr}\left(v_{i}=1 \mid V=1\right)=\operatorname{Pr}\left(v_{i}=0 \mid V=0\right)=p>1 / 2$, and $\operatorname{Pr}\left(v_{i}=1 \mid V=0\right)=\operatorname{Pr}\left(v_{i}=0 \mid V=1\right)=$ $1-p<1 / 2$ before they arrive on the market. Uninformed traders trade for liquidity reasons and don't have any information about either the quality of the company's technology $W$ or the management strength $V$. We assume that the uninformed traders choose to buy or sell with a fixed probability.

The market maker is risk-neutral and competitive. He observes the trading history $H_{t}$ and sets up different prices for the incoming buy and sell order. Ask price: $A_{t}=E\left(Y \mid H_{t}, h_{t}=B u y\right)$, Bid price: $B_{t}=E\left(Y \mid H_{t}, h_{t}=S e l l\right)$.

The market price can be written as $p=E\left(Y \mid H_{t}\right) . H_{t}=\left\{h_{1}, h_{2}, \ldots \ldots, h_{t-1}\right\}$ is the trading history just before the trade at time $t . h_{i}$ could be buy, sell, or not trade. Here we assume that market maker can observe agents who come to the market and decide not to trade. While the arrival of an agent who does not trade might not be observable in 
reality, total volume is observable and the arrival of many no-trade decisions reduces volume. Also, the market maker can observe the time between orders. If the time between two orders is significantly longer than the average, it is very possible that some agent may arrive and decide not to trade. The bid-ask spread is $\Sigma_{t}=A_{t}-B_{t}$. We define the market maker's assessed distribution on the quality of technology $W$ and the management strength $V$ as $\phi_{w}^{t}=P\left(W=w \mid H_{t}\right)$ and $\pi_{v}^{t}=P\left(\mathrm{~V}=v \mid H_{t}\right)$.

\section{Herd Behavior in the Single Risky Asset Market}

The key idea in an informational cascade is that no new information reaches the market when informed traders with different private signals choose the same action. Similarly, we can introduce the concept of an informational block that happens to some agents if the trade just presented will not change their expectation of asset value. The formal definition is as follows. We should note that it is possible that at the same time an informational block occurs to one type of market participants while no informational block happens to other types.

Definition 1, Informational Block: Informational Block happens to a particular type of market participants when $E\left(Y \mid H_{t}\right.$, Agent's Information $)=E\left(Y \mid H_{t}, h_{t}\right.$, Agent's Information $)$ and $\operatorname{Pr}\left(Y=E\left(Y \mid H_{t}\right.\right.$, Agent's Information $\left.)\right)<1$.

The term "herd behavior" is used to describe the phenomenon that informed traders make the same decision to buy or sell even when their private signals are different. In this model, it means that the informed traders with varying signals of $Y, y_{i}=v_{i} w_{i}$, are trading the same direction. The definition of herding is focused on the agent's behavior pattern.

Definition 2, Herd behavior: We define that herd behavior occurs in the market at time $\mathrm{t}$ if (Herd Buy) $\operatorname{Pr}\left(h_{t}=\right.$ Buy $\mid H_{t}$, Agent $=$ Informed $)=1$, or $($ Herd Sell $) \operatorname{Pr}\left(h_{t}=\right.$ Sell $\mid H_{t}$, Agent $=$ Informed $)=1$.

We assume that no herd behavior occurs at time $t=0$ and provide the following propositions about the existence of the herd behavior.

Proposition 1: When no uninformed traders exist, i.e., $\mu=1$, herd behavior can never happen in market equilibrium.

(Proof: See Appendix)

Proposition 2: when uninformed traders exist, i.e., $\mu<1$, herd behavior happens with a strictly positive possibility. What's more, herding in the wrong direction also happens with a strictly positive possibility.

\section{(Proof: See Appendix)}

Propositions 1 and 2 show that the information asymmetry and the trading activity of uninformed traders together can lead to an "improper" market price. We think of the price as "proper" if it can make informed traders with different private signals choose different actions in the market and make the trading orders submitted informative. The "improper" market price loses its function of separating informed traders with different private signals. In our model, it results in that all the informed traders choosing the same action regardless of their own private signals. This is exactly a herd behavior. We provide a numerical example to further illustrate how that herd behavior can happen in the following paragraph.

Example 1: We consider an economy in which the proportion of informed traders is $\mu=0.17$, with the remaining $83 \%$ of traders being uninformed. The informed trader receives a perfect signal $w$ about $W$ and a noisy signal $v$ about $V$. The accuracy of the informed trader's signal on $V$ is $\operatorname{Pr}(V=v)=0.517$. The prior belief is $\operatorname{Pr}(W=1)=0.98, \operatorname{Pr}(W=0)=0.02$, and $\operatorname{Pr}(V=1)=\operatorname{Pr}(V=0)=0.50$. For the sake of simplicity, in this example we assume that the uninformed trader's decision won't be affected by the market price. In detail, we assume that any uninformed trader will buy with probability 0.4 , sell with probability 0.4 , and choose not to trade with probability 0.2 , regardless of the market price. Suppose the realized state of $W$ is $W=1$. Hence, all the informed traders know this by receiving signal $w=1$, and they receive different signals on $V$. We call the signal $w=1 \& v=1$ a positive signal and $w=1 \& v=0$ a negative signal. We consider the case of consecutive sell orders that could lead to herd buy. To the market maker, a sell order could come from the informed trader with either a strong-negative signal $w=0$ or a weak-negative signal $w=1 \& v=0$, since he does not know $W=1$. Hence, he needs to assign positive probabilities on both signals. As we have discussed above, the strong-negative signal will create a larger downward price pressure than the weak-negative signal, since $w=0$ means that the asset payoff is absolutely zero. As soon as the ask price is lower than a critical value, herd behavior occurs. The specific pricing formulas are listed in the proof of proposition 3 in the Appendix.

Table 1 shows the details of the market facing consecutive sell orders. Before period 1, the price is equal to the unconditional expectation, i.e. $P_{1}=0.49$. The market maker sets the ask and bid price as $A_{1}=0.4955$ and 
$B_{1}=0.4846$. The informed trader with a negative signal have $E(Y \mid w=1, v=0)=0.4830<B_{1}$. Therefore, the informed trader with a weak-negative signal will sell according to his private signal, and there is no herd behavior in period 1 .

Table 1. Market facing consecutive sell orders with a rational market maker

\begin{tabular}{llll}
\hline Time & $\mathrm{t}=1$ & $\mathrm{t}=2$ & $\mathrm{t}=3$ \\
\hline Trading History & $\mathrm{N} / \mathrm{A}$ & $\mathrm{t}=1$ : Sell & $\mathrm{t}=1$ : Sell; $\mathrm{t}=2$ Sell \\
$\mathrm{E}(\mathrm{Y} \mid \mathrm{H}(\mathrm{t}), \mathrm{w}=1, \mathrm{v}=0)$ & 0.4830 & 0.4795 & 0.4795 \\
$\mathrm{~A}(\mathrm{t})$ & 0.4955 & 0.4905 & 0.3539 \\
$\mathrm{~B}(\mathrm{t})$ & 0.4846 & 0.3205 & 0.2694 \\
Ask-Bid Spread & 0.0109 & 0.1700 & 0.0845 \\
Decision of the informed trader with signal $(\mathrm{w}=1 \& \mathrm{v}=0)$ & Sell & Not Trade & Buy \\
Herd & No & No & Yes \\
Informational Block to the Informed Trader & No & Yes for Sell order, No for Buy order & Yes \\
\hline
\end{tabular}

After the first sell order in period 1, the market maker updates the price as $A_{2}=0.4905$ and $B_{2}=0.3205$. Since $A_{2}>E\left(Y \mid H_{2}=(S), w_{2}=1 \& v_{2}=0\right)=0.4795>B_{2}$, the informed trader will not trade instead of selling when he receives the negative signal. Herd behavior does not occur, since informed traders with different signals act differently. We should, however, notice that there are two important features for this period. The first feature is that the sell order is now uninformative to the informed trader, since he is able to conclude that it can only come from the uninformed trader because even the informed trader with a weak-negative signal will not sell. The second feature is that the price drops more quickly than before. In this period, facing a sell order, the rational market maker can also infer that the informed trader with a weak-negative signal $w_{2}=1 \& v_{2}=0$ will not sell. Therefore, to the market maker, the decision to sell, if it is from the informed, come only from the informed trader with a strong-negative signal $w_{2}=0$, which indicates that the asset's payoff is surely zero. Therefore, this sell in the second period will affect the price more substantially than the sell order during the first period.

After the first and second sell, the market maker sets the price as $A_{3}=0.3539$ and $B_{3}=0.2694$. It will cause a herd buy, since $E\left(Y \mid H_{3}=(S, S), w_{2}=1 \& v_{2}=0\right)=0.4700>A_{3}$ and every informed trader will buy regardless of his private signal. We should note that up to now we only assume the realization of $W$ is $W=1$ and impose no restriction on $V$. Therefore, $V$ could be either 1 or 0 . If $V=0$, we have $Y=W V=0$. It implies that the herd buy is going in the wrong direction. Hence this example shows not only the existence of herd behavior, but also the possibility of herding in the wrong direction.

During the herd buy, the probability that the incoming order is a buy order is large, since all informed traders will buy. Hence, the accumulation of excessive buy orders will alleviate the market maker's concern of $W=0$, and make the price rebound. This post-crash rebound will only happen when $W=1$. If $W=0$, no matter how low the price is, as long as it is greater than zero, the informed traders will stick to sell since they know that $Y=W V=0$. Correspondingly, no herd buy will happen as the rebound after crash.

Figure 1 depicts the evolution path from the normal phase to the herd phase. Phase 1 is the normal phase where the informed traders with different signals will act differently. Informed traders with positive signals will buy, and those with negative signals will sell. Hence trades provide information to both the market maker and future informed traders. When the expectation of informed traders with negative signal falls into the bid-ask spread, we enter Phase 2, the transition phase. The informed traders with weak-negative signals will choose not to trade rather than choosing to buy or sell. Therefore, at least some orders, like the sell order in this example, will become uninformative to the informed trader. We can call it as a "half-informational block" to the informed trader. Phase 3, the herd phase, happens when all the informed traders' expectations concentrate on one side of the price. A herd buy occurs in this example. The trading is not informative to the informed trader at all, but the market maker can still obtain information from the order flow. Thus, to the informed trader, an informational block happens when there is a herd behavior. 


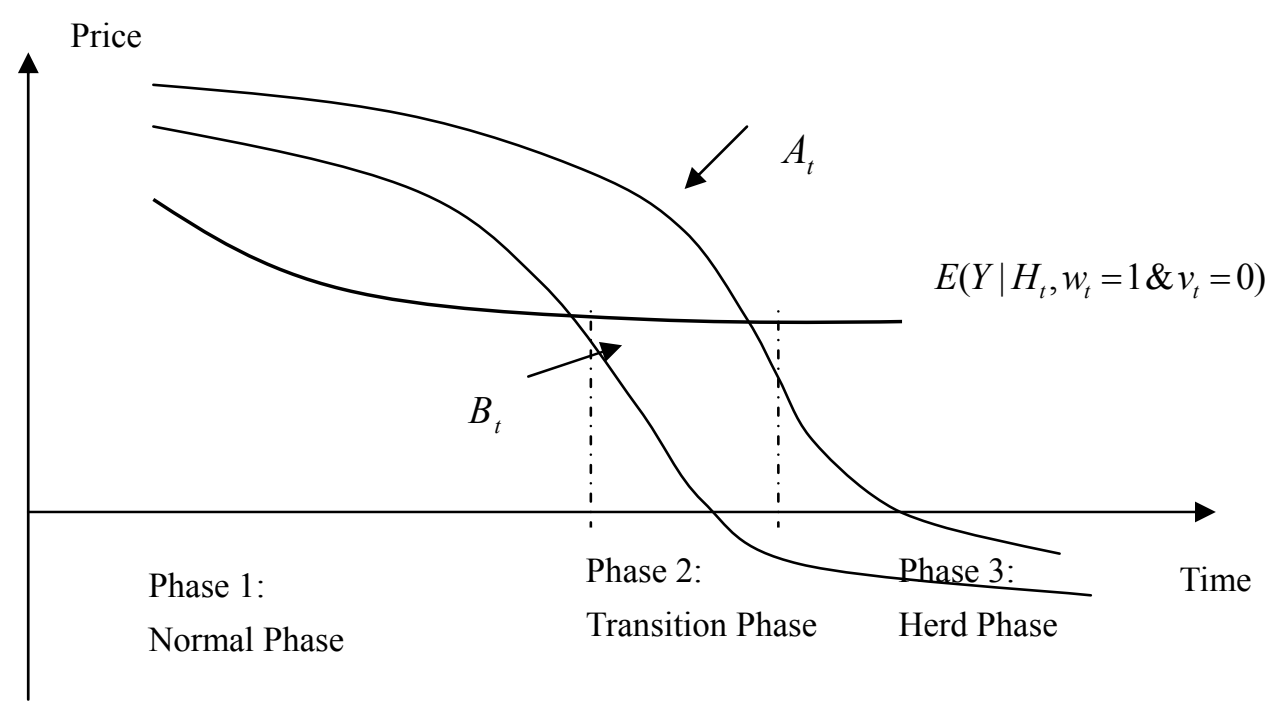

Figure 1. Evolution to herd with consecutive sell orders

There are several interesting features in this transition phase. The bid-ask spread becomes larger. The market depth decreases, because the trade in the transition phase has stronger price impact power. If the trade presented in the transition phase is unfavorable, market price could change rapidly and the herd behavior occurs. As a formal description, we provide the following definition and propositions.

Definition 3, Transition Phase: We define that the market is in the transition phase if some informed traders' expectation of the asset payoff is within the bid-ask spread, that is, $B_{t}<E\left(Y \mid H_{t}\right.$, Informed Agent's Singal $)<A_{t}$.

Proposition 3: If we assume that there is only one risky asset traded in the market, the market must enter and stay in the transition phase for at least one period before the herd behavior occurs. In other words, it cannot jump from the normal phase to the herd phase directly.

\section{(Proof: See Appendix)}

Proposition 4: The bid-ask spread in transition phase is larger than both the spread in the normal phase and the spread in the herd phase, holding everything else equal.

\section{(Proof: See Appendix)}

Proposition 3 validates the existence of transition phase. Since the market maker is risk-neutral and the spread is totally determined by the adverse selection cost in our model, Proposition 4 implies that the adverse selection is most severe in the transition phase. As we have discussed above, in our model there are three types of signals for the informed trader: the strong-negative signal $(w=0)$, the weak-negative signal $(w=1 \& v=0)$, and the positive signal $(w=1 \& v=1)$. In the normal phase, the sell could come from either the informed trader with the strong-negative signal or the informed trader with the weak-negative signal, and the buy is from the informed trader with the positive signal. When the market enters the transition phase, the market maker knows that the sell order could come only from the informed trader with the strong-negative signal, while the meaning of buy order remains the same. Hence, the bid price will drop quickly with the incoming sell orders and the bid-ask spread will increase. In the herd phase, the information content of a sell is the same as in the transition phase, while the information content of a buy order is weakened due to the herd buy. Therefore, the bid-ask spread in the herd phase will be smaller than the spread in the transition phase.

Proposition 5: Considering the magnitude of price adjustment caused by the trade just-presented, that is, $\Delta A_{t}=\left|A_{t+1}-A_{t}\right|$ and $\Delta B_{t}=\left|B_{t+1}-B_{t}\right|$, we have $\Delta A_{t}^{T} \geq \Delta A_{t}^{N}, \Delta A_{t}^{T} \geq \Delta A_{t}^{H}$, and $\Delta B_{t}^{T} \geq \Delta B_{t}^{H}$, when holding everything else equal. (The superscripts of $T, N, H$ are correspondingly the symbol of Transition phase, Normal phase, and Herd phase.)

(Proof: See Appendix)

Proposition 6: If the agent just arriving at time $t$ chooses not to trade, there is no price change in the normal phase and the herd phase. In the transition phase, however, the price will change. The bid price will increase 
when $\phi_{1}^{t}<\frac{g(1-\delta)}{\delta+g-\delta g}$ and decrease when $\phi_{1}^{t}>\frac{g(1-\delta)}{\delta+g-\delta g}$. The ask price will increase when $\phi_{1}^{t}<\frac{\mu p(1-h)+\gamma g}{\gamma(h+g-1)}$ and decrease when $\phi_{1}^{t}>\frac{\mu p(1-h)+\gamma g}{\gamma(h+g-1)}$.

$\left(\delta, g\right.$ and $h$ are all functions of $\pi_{1}^{t}$ and $\pi_{0}^{t} \cdot \delta=\frac{\mu \pi_{0}^{t}(2 p-1)}{\mu\left[p \pi_{0}^{t}+(1-p) \pi_{1}^{t}\right]+\gamma}, g=\frac{\mu\left[p \pi_{0}^{t}+(1-p) \pi_{1}^{t}\right]}{\mu\left[p \pi_{0}^{t}+(1-p) \pi_{1}^{t}\right]+\gamma}$ and $\left.h=\frac{\mu\left[(1-p) \pi_{0}^{t}+p \pi_{1}^{t}-(2 p-1) \delta \pi_{1}^{t}\right]+\gamma}{(1-\delta)\left\{\mu\left[(1-p) \pi_{0}^{t}+p \pi_{1}^{t}\right]+\gamma\right\}}.\right)$

(Proof: See Appendix)

Proposition 5 shows that usually in the transition phase the price change caused by the incoming trade order is likely to be larger than or equal to that in the normal phase and herd phase when we hold everything else equal. In other words, market price is more sensitive to the trades presented in the transition phase. In addition, Proposition 6 indicates that, even facing an agent who chooses not to trade, the market maker will change his quotes in the transition phase. In the normal phase and the herd phase, only uninformed traders will choose not to trade. Every informed trader will buy or sell according to his own signal. However, in the transition phase, the informed trader with weak-negative signal $w=1 \& v=0$ will not trade, since his expectation of the asset value is within the bid-ask spread. Hence, the decision of not trading becomes informative in the transition phase and is able to changes the market maker's quotes.

In this section we have constructed a model which a market crash may happen even if the market maker and all the informed traders are fully rational. Whether a rebound can happen after the crash depends on the value of $W$. Also, we find that there exists a transition phase between the normal phase and herd phase. The landmark of the transition phase is the deterioration of market liquidity. Proposition 4 shows that the bid-ask spread width increases in the transition phase. Proposition 5 shows that the price impact power of incoming orders is stronger in the transition phase. The notion that market liquidity is low in the transition phase, which is associated with higher non-trading probability, is consistent with findings in recent empirical microstructure studies. For example, Lesmond (2005) and Bekaert, Harvey and Lundblad (2007) suggest that large number of non-trading days implies low liquidity in the stock market. Kang and Zhang (2012) also show that the proportion of non-trading days is a good measure of liquidity in emerging equity markets.

\section{Contagion}

Finance literature shows that bank runs could be contagious. So how about the "stock run"? Could the herd behavior be contagious in the markets with different assets whose values are correlated? We believe that as long as traders and market makers are able to observe the history of trade in other markets, the correlation between the asset values can cause herd behavior to be contagious. More precisely, we define contagious spillover as the following: herd behavior in one asset market would not occur if agents in this market were not allowed to observe the trade history of other asset markets.

Definition 4: We define that there is a contagious spillover from asset B to asset $\mathrm{A}$ at time $t$ if

(Herd Buy) $\operatorname{Pr}\left(h_{t}^{A}=\right.$ Buy $\mid H_{t}^{A}, H_{t}^{B}$, Agent $=$ Informed $)=1$, while $\operatorname{Pr}\left(h_{t}^{A}=\right.$ Buy $\mid H_{t}^{A}$, Agent $=$ Informed $)<1$ or (Herd Sell) $\operatorname{Pr}\left(h_{t}^{A}=\right.$ Sell $\mid H_{t}^{A}, H_{t}^{B}$, Agent $=$ Informed $)=1$, while $\operatorname{Pr}\left(h_{t}^{A}=\right.$ Sell $\mid H_{t}^{A}$, Agent $=$ Informed $)<1$.

We then present an example of how contagious spillover happens between two asset markets. In this example, what happens in one market not only triggers herd behavior in another market, but also actually accelerates the pace toward herd behavior in that market.

Example 2: We assume that now in the economy there are two risky assets: asset A and B. The payoff of asset A is $Y_{A}=W V_{A}$, and the payoff of asset B is $Y_{B}=W V_{B}$. We suppose $\operatorname{Corr}\left(V_{A}, V_{B}\right)=0$, which means $W$ is the only link between $Y_{A}$ and $Y_{B}$. This setup can be applied to many realistic situations. For example, A and B belongs to the same industry that use the same set of production technologies, while they have difference management strength. In the economy, the proportion of informed traders in both markets is $\mu_{A}=\mu_{B}=0.17$. The informed trader receives a perfect signal $w$ about $W$ and a noisy signal $v$ about $V$. The accuracy of the informed trader's signal on $V$ is different: $\operatorname{Pr}\left(V_{A}=v_{A}\right)=0.557$ and $\operatorname{Pr}\left(V_{B}=v_{B}\right)=0.517$. The prior belief is $\operatorname{Pr}(W=1)=0.98, \operatorname{Pr}(W=0)=0.02$, $\operatorname{Pr}\left(V_{A}=1\right)=\operatorname{Pr}\left(V_{A}=0\right)=0.50, \operatorname{Pr}\left(V_{B}=1\right)=\operatorname{Pr}\left(V_{B}=0\right)=0.50$. We still assume that any uninformed trader coming to the market will buy with probability 0.4 , sell with probability 0.4 , and choose not to trade with probability 0.2 , 
regardless of the market price. Suppose the realized state of $W$ is $W=1$. Hence all the informed traders know this and will receive different signals on $V_{A}$ or $V_{B}$. We call the signal $w=1 \& v=1$ a positive signal and $w=1 \& v=0$ a negative signal. Table 2 shows the response of asset market A alone facing consecutive sells. Asset market $\mathrm{B}$ alone is actually what we have discussed in Example 1. Table 3 shows what will happen when these two markets face consecutive sell orders together.

In this example, herd behavior is less likely to happen in market $\mathrm{A}$, because the precision of private signals in $V_{A}$ is higher than the precision of private signals in $V_{B}$. From Table 2 showing the details of market A facing consecutive sells alone, we can see that we need 10 consecutive sell orders to push market A into the transition phase, and an extra 11th sell to enable the herd buy in the market. Compared with market B alone which needs only 3 consecutive sells to enter the transition phase and an extra 4th sell to trigger the herd buy as described in Table 1 in Section 3, we find that even a slight increase in the signal precision $(\operatorname{Pr}(V=v)=0.517 \rightarrow 0.557)$ will reduce the possibility of herd behavior greatly.

Table 2. Market A alone facing consecutive sell orders

\begin{tabular}{lllllll}
\hline Time & $\mathrm{t}=1$ & $\mathrm{t}=2$ & $\mathrm{t}=3$ & $\mathrm{t}=4$ & $\mathrm{t}=5$ & $\mathrm{t}=6$ \\
\hline $\mathrm{A}(\mathrm{t})$ & 0.5037 & 0.4907 & 0.4775 & 0.4640 & 0.4504 & 0.4364 \\
$\mathrm{~B}(\mathrm{t})$ & 0.4767 & 0.4631 & 0.4492 & 0.4351 & 0.4206 & 0.4057 \\
Ask-Bid Spread & 0.0270 & 0.0276 & 0.0282 & 0.0290 & 0.0298 & 0.0307 \\
$\mathrm{E}(\mathrm{Y} \mid \mathrm{H}(\mathrm{t}), \mathrm{w}=1, \mathrm{v}=0)$ & 0.4430 & 0.4316 & 0.4202 & 0.4089 & 0.3977 & 0.3867 \\
Decision of the informed trader with the weak-negative signal & Sell & Sell & Sell & Sell & Sell & Sell \\
Herd & No & No & No & No & No & No \\
\hline Time & $\mathrm{t}=7$ & $\mathrm{t}=8$ & $\mathrm{t}=9$ & $\mathrm{t}=10$ & $\mathrm{t}=11$ & \\
\hline $\mathrm{A}(\mathrm{t})$ & 0.4222 & 0.4077 & 0.3928 & 0.3776 & 0.2692 \\
$\mathrm{~B}(\mathrm{t})$ & 0.3905 & 0.3749 & 0.3588 & 0.2373 & 0.1569 \\
Ask-Bid Spread & 0.0317 & 0.0328 & 0.0340 & 0.1403 & 0.1123 \\
$\mathrm{E}(\mathrm{Y} \mid \mathrm{H}(\mathrm{t}), \mathrm{w}=1, \mathrm{v}=0)$ & 0.3757 & 0.3649 & 0.3542 & 0.3436 & 0.3436 \\
Decision of the informed trader with the weak-negative signal & Sell & Sell & Sell & Not Trade & Buy \\
Herd & No & No & No & No & Yes \\
\hline
\end{tabular}

Table 3. Markets A and B facing consecutive sell orders together

\begin{tabular}{lllll}
\hline Time & $\mathrm{t}=1$ & $\mathrm{t}=2$ & $\mathrm{t}=3$ & $\mathrm{t}=4$ \\
\hline $\begin{array}{l}\text { Where the trade actually happens } \\
\text { Trading History }\end{array}$ & $\mathrm{A}$ & $\mathrm{A}$ & $\mathrm{B}$ \\
& $\mathrm{N} / \mathrm{A}$ & $\mathrm{t}=1$ : Sell in A & $\begin{array}{l}\mathrm{t}=1 \text { : Sell in A; } \mathrm{t}=2 \text { : Sell in } \\
\mathrm{A}\end{array}$ & $\begin{array}{l}\mathrm{A}=1 \text { : Sell in A; } \mathrm{t}=2 \text { : Sell in A; } \\
\mathrm{t}=3 \text {; Sell in B }\end{array}$ \\
\hline Market A & & & & \\
\hline $\mathrm{A}(\mathrm{t})$ & 0.5037 & 0.4907 & 0.4775 & 0.3409 \\
$\mathrm{~B}(\mathrm{t})$ & 0.4767 & 0.4631 & 0.4492 & 0.2026 \\
$\mathrm{E}(\mathrm{Y} \mid \mathrm{H}(\mathrm{t}), \mathrm{w}=1, \mathrm{v}=0)$ & 0.4430 & 0.4316 & 0.4202 & 0.4202 \\
Decision of the informed trader with the & Sell & Sell & Sell & Buy \\
weak-negative signal & & & & \\
Herd & No & No & No & Yes \\
\hline Market B & & & & 0.3552 \\
\hline A $(\mathrm{t})$ & 0.4955 & 0.4939 & 0.4920 & 0.2109 \\
$\mathrm{~B}(\mathrm{t})$ & 0.4846 & 0.3228 & 0.3212 & 0.4800 \\
$\mathrm{E}(\mathrm{Y} \mid \mathrm{H}(\mathrm{t}), \mathrm{w}=1, \mathrm{v}=0)$ & 0.4830 & 0.4830 & 0.4830 & Buy \\
Decision of the informed trader with the & Sell & Not Trade & Not Trade & Yes \\
weak-negative signal & & & & No \\
Herd & No & No & & \\
\hline
\end{tabular}

When these two markets with different signal precisions coexist and operate together, herd behavior could become contagious and spread from the market with low precision signals to the market with high precision signals. The resistance to herd behavior in the market with high precision signals is undermined by its link with the market having low precision signals. This is exactly what we see in Table 3. After the 1st and 2nd sell in asset A, the 3rd sell in asset B is enough to send both asset markets A and B into herd buy directly. The reason here is that the 1st and 2nd sells in asset market A push market B into the transition phase. As we have discussed 
in Section 3, the market in the transition phase is very sensitive. Therefore, the 3rd sell in asset market B not only enables the herd in market B itself but also triggers herd behavior in market A since the market maker's belief of $W=0$ is enhanced greatly. Here what happens in the transition phase in one asset market not only substantially impacts this market itself, but also has profound influence on other asset markets linked with this market. In other words, the market with low precision of signals is more vulnerable to herd behavior. This vulnerability is contagious to other linked markets with high information precision.

When we interpret $W$ as the firm's production technology strength, the contagion would like to happen at the industry level, since firms in the same industry tend to adopt the same or similar production technology. Little work has been done about the intra-industry contagion phenomenon and the empirical implication of this section remains to be tested in future research. Another potential application of this model is the cross-country examples. For example, in a regional financial crisis, a crash in one country's market can lead to turmoil in its neighboring countries, as investors worry about the overall financial stability of all the countries in the region.

\section{Conclusion}

In this paper we develop a rational model explaining the mechanism of market crash and the herd behavior in financial markets. We find a transition phase exists between the normal phase and the herd phase. During the transition phase, the market liquidity deteriorates, marked by larger bid-ask spread and stronger price impact power. The trades presented in the transition phase have substantial influence on the market price. Whether a rebound can happen after the crash depends on the nature of the world, that is, the value of $W$ in our model. If $W=1$, a herd buy could happen as the rebound. If $W=0$, the market price will stay stable at the low level with no rebound. Then we extend our analysis into the multiple markets situation to examine the mechanism of contagion. We assume that the precision of the informed trader's signal is different in different markets. High precision will make herd behavior unlikely since the informed will more likely follow his signal than follow the trade history. If we mix the high precision and low precision markets together, we find that herd behavior could be contagious from one market to another. In our example, the sell in the transition phase in the low precision market not only brings this market into herd phase, but also triggers herd behavior in the high precision market.

The theoretical predictions from this model can be tested by empirically examining whether liquidity will dry up during the market crisis, and whether stock return comovement and liquidity commonality will increase in such period. These empirical tests can be conducted either at the industry level or at a broad market level. It also would be interesting to conduct more research into the features of the transition phase and the contagion part. For example, future empirical research work can examine if there is any contagion of the financial crisis cross countries in the same geographic region.

One policy recommendation suggested by this study is that during a financial crisis, the company with weak fundamentals might experience the stock price decrease first. Then, because of the contagion mechanism introduced in this paper, even company with solid fundamentals could also witness rapid stock price decline. Therefore, it highlights the importance of implementing market stabilization procedures to prevent further market panic movements and reduce the impact from financial crisis.

\section{Acknowledgements}

I am grateful for comments from Christopher Avery, Antonio Bernardo, Sushil Bikhchandani, Carla Hayn, Mark Junkunc, Jun Liu, Avanidhar Subrahmanyam, and Peter Zemsky, as well as the seminar participants at Eastern Finance Association conference.

\section{References}

Avery, C., \& Zemsky, P. (1998). Multidimensional uncertainty and herd behavior in financial markets. American Economics Review, 88, 724-748.

Banerjee, A. (1992). A simple model of herd behavior. Quarterly Journal of Economics, 107, 797-818. http://dx.doi.org/10.2307/2118364

Bekaert, G., Harvey, C., \& Lundblad, C. (2007). Liquidity and expected returns: Lessons from emerging markets. Review of Financial Studies, 20, 1783-1831. http://dx.doi.org/10.1093/rfs/hhm030

Bikhchandani, S., Hirshleifer, D., \& Welch, I. (1992). A theory of fads, fashion, custom, and cultural change as informational cascades. Journal of Political Economy, 100, 992-1026. http://dx.doi.org/10.1086/261849

Bulow, J., \& Klemperer, P. (1994). Rational Frenzies and Crashes. Journal of Political Economy, 102, 1-24. http://dx.doi.org/10.1086/261919

Easley, D., \& O’Hara, M. (1992). Time and the process of security price adjustment. Journal of Finance, 47, 
576-605. http://dx.doi.org/10.1111/j.1540-6261.1992.tb04402.x

Glosten, L., \& Milgrom, P. (1985). Bid, ask and transaction prices in a specialist market with heterogeneously informed traders. Journal of Financial Economics, 14, 71-100. http://dx.doi.org/10.1016/0304-405X(85)90044-3

Grinblatt, M., Titman, S., \& Wermers, R. (1995). Momentum investment strategies, portfolio performance, and herding: A study of mutual fund behavior. American Economic Review, 85, 1088-1105.

Kang, W., \& Zhang, H. (2012). Measuring liquidity in emerging markets. Working Paper.

Lao, P., \& Singh, H. (2011). Herding behaviour in the Chinese and Indian stock markets. Journal of Asian Economics, 22, 495-506. http://dx.doi.org/10.1016/j.asieco.2011.08.001

Lee, I. (1998). Market crashes and informational avalanches. Review of Economic Studies, 65, 741-759. http://dx.doi.org/10.1111/1467-937X.00066

Lesmond, D. (2005). Liquidity of emerging markets. Journal of Financial Economics, 77, 411-452. http://dx.doi.org/10.1016/j.jfineco.2004.01.005

Nofsinger, J., \& Sias, R. (1999). Herding and feedback trading by institutional and individual investors. Journal of Finance, 54, 2263-2295. http://dx.doi.org/10.1111/0022-1082.00188

Ouarda, M., Bouri, A., \& Bernard, O. (2013). Herding behavior under markets condition: Empirical evidence on the European financial markets. International Journal of Economics and Financial Issues, 3, 214-228.

Romer, D. (1993). Rational asset-price movements without news. American Economic Review, 83, 1112-30.

Scharfstein, D., \& Jeremy, S. (1990). Herd behavior and investment. American Economic Review, 80, 465-479.

Subrahmanyam, A., \& Titman, S. (2001). Feedback from stock prices to cash flows. Journal of Finance, 56, 2389-2413. http://dx.doi.org/10.1111/0022-1082.00409

Trueman, B. (1994). Analyst forecasts and herding behavior. Review of Financial Studies, 7, 97-124. http://dx.doi.org/10.1093/rfs/7.1.97

\section{Appendix}

\section{Proof of Proposition 1}

The proof of proposition 1 is straightforward. Given that all the traders are informed and know $W$ for sure, the first buy order will let the market maker realize $W=1$, and we come back to the single-dimensional uncertainty case where no herd behavior could happen. If the buy order never comes, it proves that the true state is $W=0$. The market maker is correct to always decrease the price until it approaches zero.

\section{Proof of Proposition 2}

Suppose $W=1$ and no herd behavior occurs at the beginning. Every sell order will increase $\phi_{0}^{t}$, the market maker's belief of $W=0$. Because of the existence of uninformed traders, any trade history is possible. So we consider the situation of consecutive sell orders, that is, every incoming order is sell. In this case, as $t$ increases, $\phi_{0}^{t}$ will increase. As we know, as $t \rightarrow \infty, \phi_{0}^{t} \rightarrow 1$. In other words, if we need $\phi_{0}^{t}$ greater than a positive number less than 1 , we can do this by assuming finite consecutive sell orders.

Under the assumption that $W=1$, in order to enter the transition phase we need:

$$
\begin{gathered}
E\left(Y \mid w=1, v=0, H_{t}\right)=\frac{(1-p) \pi_{1}^{t}}{(1-p) \pi_{1}^{t}+p \pi_{0}^{t}}>\frac{\mu \phi_{1}^{t}(1-p) \pi_{1}^{t}+\gamma \phi_{1}^{t} \pi_{1}^{t}}{\mu\left[\phi_{0}^{t}+\phi_{1}^{t}\left(p \pi_{0}^{t}+(1-p) \pi_{1}^{t}\right)\right]+\gamma}=B_{t} \\
\left.\Leftrightarrow(1-p)\left\{\mu\left[\phi_{0}^{t}+\phi_{1}^{t}\left(p \pi_{0}^{t}+(1-p) \pi_{1}^{t}\right)\right]+\gamma\right\}>[1-p) \pi_{1}^{t}+p \pi_{0}^{t}\right]\left[\mu \phi_{1}^{t}(1-p)+\gamma \phi_{1}^{t}\right] \\
\Leftrightarrow(1-p)\left\{\mu\left[\lambda^{t}+\left(p \pi_{0}^{t}+(1-p) \pi_{1}^{t}\right)\right]+\gamma\right\}>\left[(1-p) \pi_{1}^{t}+p \pi_{0}^{t}\right][\mu(1-p)+\gamma]\left(\text { define } \lambda^{t}=\phi_{0}^{t} / \phi_{1}^{t}\right) \\
\Leftrightarrow(1-p) \mu \lambda^{t}+(1-p) \gamma>\gamma\left[(1-p) \pi_{1}^{t}+p \pi_{0}^{t}\right] \Leftrightarrow \lambda^{t}>\frac{\gamma\left[(1-p) \pi_{1}^{t}+p \pi_{0}^{t}\right]}{(1-p) \mu}-\frac{\gamma}{\mu}
\end{gathered}
$$

Since $(1-p) \pi_{1}^{t}+p \pi_{0}^{t}<p \pi_{1}^{t}+p \pi_{0}^{t}=p$, as long as $\lambda^{t}>\frac{\gamma p}{(1-p) \mu}-\frac{\gamma}{\mu}=\frac{(2 p-1) \gamma}{(1-p) \mu}$, i.e., $\phi_{0}^{t}>\frac{\gamma(2 p-1)}{\mu(1-p)+\gamma(2 p-1)}$, the inequality above is valid. Because $0<\frac{\gamma(2 p-1)}{\mu(1-p)+\gamma(2 p-1)}<1$ 
we can assume that after finite $n$ sells we will have $E\left(Y \mid w=1, v=0, H_{t}\right)>B_{t}$ and enter the transition phase.

Now, suppose that at $t=n$ we enter the transition phase. If $E\left(V \mid w=1, v=0, H_{n}\right)>A_{n}$, herd behavior occurs directly. If $E\left(V \mid w=1, v=0, H_{n}\right)<A_{n}$, we keep assuming that all the trades arriving after $t=n$ are still sells. In the transition phase, the market maker can set up the ask price in the following way: $A_{t}=\left(1-\phi_{0}^{t}\right) A^{\prime}{ }_{n}$ given $t>n$, since the market maker knows that under the assumption $W=1$, every sell after $t=n$ is uninformative. $A_{n}^{\prime}$ is the ask price the market maker would ask at $t=n$ if he knew $W=1$, $1>A_{n}^{\prime}>E\left(V \mid w=1, v=0, H_{n}\right)$. The informed trader keeps his expectation unchanged since he also knows that with signal $w=1$ every sell after $t=n$ is uninformative. Hence, we need $A_{t}=\left(1-\phi_{0}^{t}\right) A_{n}^{\prime}<E\left(V \mid w=1, v=0, H_{n}\right)$ to enable herd behavior. This is equal to $\phi_{0}^{t}>1-E\left(V \mid w=1, v=0, H_{n}\right) / A_{n}^{\prime}$. The right side is a finite positive number smaller than 1 . Hence, we can assume that after extra $m$ sells, at $t=m+n$, we have $\phi_{0}^{t}>1-E\left(V \mid w=1, v=0, H_{n}\right) / A_{n}^{\prime}$, which means that a herd buy occurs. We can furthermore assume $V=0$, which means that the herd buy above is actually going in the wrong direction. Therefore, not only herd behavior will happen, but also herding in the wrong direction will happen with strict positive probability.

\section{Proof of Proposition 3}

First, let us use $A_{t}^{N}$ and $B_{t}^{N}$ to represent the ask and bid price in normal phase. Similarly, $A_{t}^{T}$ and $B_{t}^{T}$ are the ask and bid price in transition phase, and $A_{t}^{H}$ and $B_{t}^{H}$ are the ask and bid price in herd phase. Now we show that if at time $t$ the market is in normal phase, it is impossible for the market to jump into herd phase directly at $t+1$. We know $E\left(V \mid w_{t}=1, v_{t}=0, H_{t}\right)<B_{t}^{N}$ from the assumption that the market is in normal phase at time $t$. If the market jumped into herd phase at $t+1$, we must have not only $E\left(V \mid w_{t+1}=1, v_{t+1}=0, H_{t+1}\right)>A_{t+1}^{H}$ but also $E\left(V \mid w_{t+1}=1, v_{t+1}=0, H_{t+1}\right)>A_{t+1}^{N}$. Otherwise, the market maker would set the ask price as $A_{t+1}^{N}$ and the herd buy cannot happen.

We know that in our model only excessive sells can lead the market to herd behavior. Therefore, if the market is in the normal phase at time $t$, a buy order or a decision of not to trade cannot trigger the herd behavior, since in normal phase buy order can come from either the informed trader with signal of $w_{t}=1, v_{t}=1$ or the uninformed, and the decision of not to trade can come only from the uninformed. Hence, the only possibility for herd behavior to occur is to have a sell at time $t$. But if there is a sell at time $t$, we have $E\left(V \mid w_{t+1}=1, v_{t+1}=0, H_{t+1}\right)<E\left(V \mid w_{t}=1, v_{t}=0, H_{t}\right)$, because $H_{t+1}=\left\{H_{t}, h_{t}=\right.$ Sell $\}$ includes more negative information than $H_{t}$. We have $B_{t}{ }^{N}<A_{t+1}^{N} \quad$ from $B_{t}^{N}=E\left(V \mid H_{t}=\left\{h_{1}, \ldots, h_{t-1}\right\}, h_{t}=\operatorname{Sell}\right)$, $A_{t+1}^{N}=E\left(V \mid H_{t+1}=\left\{h_{1}, \ldots, h_{t-1}, h_{t}=S e l l\right\}, h_{t+1}=B u y\right)$, and in the normal phase, a buy from informed trader means his or her signal of $w=1 \& v=1$.

Therefore, we have $E\left(V \mid w_{t+1}=1, v_{t+1}=0, H_{t+1}\right)<E\left(V \mid w_{t}=1, v_{t}=0, H_{t}\right)<B_{t}^{N}<A_{t+1}^{N}$, which is contradictory to the requirement above for the market to jump into herd phase directly. The market must stay in the transition phase for at least one period before entering the herd phase, given there is only one risky asset traded in the market.

The pricing formulas in normal phase, transition phase and herd phase in our model are the following.

Normal Phase: $A_{t}^{N}=\frac{\mu p \pi_{1}^{t}+\gamma \phi_{1}^{t} \pi_{1}^{t}}{\mu\left[p \pi_{1}^{t}+(1-p) \pi_{0}^{t}\right]+\gamma} ; B_{t}^{N}=\frac{\mu \phi_{1}^{t}(1-p) \pi_{1}^{t}+\gamma \phi_{1}^{t} \pi_{1}^{t}}{\mu\left[\phi_{0}^{t}+\phi_{1}^{t}\left(p \pi_{0}^{t}+(1-p) \pi_{1}^{t}\right)\right]+\gamma} ;$

Transition Phase: $A_{t}^{T}=\frac{\mu p \pi_{1}^{t}+\gamma \phi_{1}^{t} \pi_{1}^{t}}{\mu\left[p \pi_{1}^{t}+(1-p) \pi_{0}^{t}\right]+\gamma} ; B_{t}^{T}=\frac{\mu^{*} 0+\gamma \phi_{1}^{t} \pi_{1}^{t}}{\mu+\gamma}=\frac{\gamma \phi_{1}^{t} \pi_{1}^{t}}{\mu+\gamma}$;

Herd Phase: $A_{t}^{H}=\frac{\mu\left[p \pi_{1}^{t}+(1-p) \pi_{0}^{t}\right] \pi_{1}^{t}+\gamma \phi_{1}^{t} \pi_{1}^{t}}{\mu\left[p \pi_{1}^{t}+(1-p) \pi_{0}^{t}\right]+\gamma} ; \quad B_{t}^{H}=\frac{\mu^{*} 0+\gamma \phi_{1}^{t} \pi_{1}^{t}}{\mu+\gamma}=\frac{\gamma \phi_{1}^{t} \pi_{1}^{t}}{\mu+\gamma}$.

But we don't rely on the specific form of these formulas to complete the proof of the existence of the transition phase. This should be a nice point since this result is robust in different model settings.

\section{Proof of Proposition 4}

The bid-ask spread in different phases are written as the following.

Normal Phase: $\Sigma_{t}^{N}=A_{t}^{N}-B_{t}^{N}=\frac{\mu p \pi_{1}^{t}+\gamma \phi_{1}^{t} \pi_{1}^{t}}{\mu\left[p \pi_{1}^{t}+(1-p) \pi_{0}^{t}\right]+\gamma}-\frac{\mu \phi_{1}^{t}(1-p) \pi_{1}^{t}+\gamma \phi_{1}^{t} \pi_{1}^{t}}{\mu\left[\phi_{0}^{t}+\phi_{1}^{t}\left(p \pi_{0}^{t}+(1-p) \pi_{1}^{t}\right)\right]+\gamma}$ 
Transition Phase: $\Sigma_{t}^{T}=A_{t}^{T}-B_{t}^{T}=\frac{\mu p \pi_{1}^{t}+\gamma \phi_{1}^{t} \pi_{1}^{t}}{\mu\left[p \pi_{1}^{t}+(1-p) \pi_{0}^{t}\right]+\gamma}-\frac{\gamma \phi_{1}^{t} \pi_{1}^{t}}{\mu+\gamma}$

Herd Phase: $\Sigma_{t}^{H}=A_{t}^{H}-B_{t}^{H}=\frac{\mu\left[p \pi_{1}^{t}+(1-p) \pi_{0}^{t}\right] \pi_{1}^{t}+\gamma \phi_{1}^{t} \pi_{1}^{t}}{\mu\left[p \pi_{1}^{t}+(1-p) \pi_{0}^{t}\right]+\gamma}-\frac{\gamma \phi_{1}^{t} \pi_{1}^{t}}{\mu+\gamma}$

$\Sigma_{t}^{T}>\Sigma_{t}^{H} \quad$ can be proven by $\Sigma_{t}^{T}>\Sigma_{t}^{H} \Leftrightarrow A_{t}^{T}>A_{t}^{H} \Leftrightarrow \mu p \pi_{1}^{t}>\mu\left[p \pi_{1}^{t}+(1-p) \pi_{0}^{t}\right] \pi_{1}^{t} \Leftrightarrow \quad p>p \pi_{1}^{t}+(1-p) \pi_{0}^{t}$ $\Leftrightarrow p>1-p$, which is obvious by $p=\operatorname{Pr}(V=v)>0.5$. And $\Sigma_{t}^{T}>\Sigma_{t}^{N}$ is the equivalent of $B_{t}^{T}<B_{t}^{N}$, which can be proven by $\mu\left[\phi_{0}^{t}+\phi_{1}^{t}\left(p \pi_{0}^{t}+(1-p) \pi_{1}^{t}\right)\right]+\gamma \quad<\mu\left[\phi_{0}^{t}+\phi_{1}^{t}\left(p \pi_{0}^{t}+p \pi_{1}^{t}\right)\right]+\gamma=\mu\left(\phi_{0}^{t}+\phi_{1}^{t} p\right)+\gamma$ $<\mu\left(\phi_{0}^{t}+\phi_{1}^{t}\right)+\gamma=\mu+\gamma$ and $\mu \phi_{1}^{t}(1-p) \pi_{1}^{t}+\gamma \phi_{1}^{t} \pi_{1}^{t}>\gamma \phi_{1}^{t} \pi_{1}^{t}$.

\section{Proof of Proposition 5}

(i) Suppose at time $t$ a buy order comes to the market. After the execution of this buy order, the market maker will adjust the price quote correspondingly. The price adjusting comes from the change of the market maker's assessed distribution on $W$ and $V$, that is, the revision of $\phi_{1}^{t}$ and $\pi_{1}^{t}$. During the normal phase and transition phase we have

$\phi_{1, N}^{t+1}=\phi_{1, T}^{t+1}=\frac{\mu+\gamma \phi_{1}^{t}}{\mu+\gamma}=\phi_{1}^{t}+\lambda \phi_{0}^{t} \quad\left(\right.$ with $\left.\lambda=\frac{\mu}{\mu+\gamma}>0\right)$ and

$\pi_{1, N}^{t+1}=\pi_{1, T}^{t+1}=\frac{\mu \pi_{1}^{t} p+\gamma \pi_{1}^{t}}{\mu\left[\pi_{1}^{t} p+\pi_{0}^{t}(1-p)\right]+\gamma}=(1+\delta) \pi_{1}^{t}$ (with $\left.\delta=\frac{\mu \pi_{0}^{t}(2 p-1)}{\mu\left[\pi_{1}^{t} p+\pi_{0}^{t}(1-p)\right]+\gamma}>0\right)$. In the herd phase we still have $\phi_{1, N}^{t+1}=\frac{\mu+\gamma \phi_{1}^{t}}{\mu+\gamma}=\phi_{1}^{t}+\lambda \phi_{0}^{t}$, but $\pi_{1, N}^{t+1}$ is the same as $\pi_{1}^{t}$, since during the herd phase the informed buy only because they receive the clear signal $w=1$, regardless of their noisy signal $v$.

We rewrite $A_{t}^{T}, A_{t}^{N}$ as $A_{t}^{T}=A_{t}^{N}=\frac{\mu p \pi_{1}^{t}+\gamma \phi_{1}^{t} \pi_{1}^{t}}{\mu\left[p \pi_{1}^{t}+(1-p) \pi_{0}^{t}\right]+\gamma}=\frac{\mu p \pi_{1}^{t}+\gamma \phi_{1}^{t} \pi_{1}^{t}}{\mu\left[(2 p-1) \pi_{1}^{t}+(1-p)\right]+\gamma}=\frac{\mu p \pi_{1}^{t}+\gamma \phi_{1}^{t} \pi_{1}^{t}}{K_{1} \pi_{1}^{t}+K_{2}} \quad$ with $K_{1}=\mu(2 p-1) \quad$ and $\quad K_{2}=\mu(1-p)+\gamma$

Hence we have $\Delta A_{t}^{T}=\Delta A_{t}^{N}=A_{t+1}^{T}-A_{t}^{T}=\frac{\mu p \pi_{1}^{t+1}+\gamma \phi_{1}^{t+1} \pi_{1}^{t+1}}{\mu\left[p \pi_{1}^{t+1}+(1-p) \pi_{0}^{t+1}\right]+\gamma}-\frac{\mu p \pi_{1}^{t}+\gamma \phi_{1}^{t} \pi_{1}^{t}}{\mu\left[p \pi_{1}^{t}+(1-p) \pi_{0}^{t}\right]+\gamma}$ $=\frac{\mu p \pi_{1}^{t+1}+\gamma \phi_{1}^{t+1} \pi_{1}^{t+1}}{K_{1} \pi_{1}^{t+1}+K_{2}}-\frac{\mu p \pi_{1}^{t}+\gamma \phi_{1}^{t} \pi_{1}^{t}}{K_{1} \pi_{1}^{t}+K_{2}}$ $=\frac{\left[\mu p(1+\delta) \pi_{1}^{t}+\gamma\left(\phi_{1}^{t}+\lambda \phi_{0}^{t}\right)(1+\delta) \pi_{1}^{t}\right]\left[K_{1} \pi_{1}^{t}+K_{2}\right]-\left[\mu p \pi_{1}^{t}+\gamma \phi_{1}^{t} \pi_{1}^{t}\right]\left[K_{1}(1+\delta) \pi_{1}^{t}+K_{2}\right]}{\left[K_{1}(1+\delta) \pi_{1}^{t}+K_{2}\right]\left[K_{1} \pi_{1}^{t}+K_{2}\right]}$

$=\frac{\left(\mu p+\gamma \phi_{1}^{t}\right) K_{2} \delta \pi_{1}^{t}+\gamma \lambda \phi_{0}^{t}(1+\delta) \pi_{1}^{t}\left(K_{1} \pi_{1}^{t}+K_{2}\right)}{\left[K_{1}(1+\delta) \pi_{1}^{t}+K_{2}\right]\left[K_{1} \pi_{1}^{t}+K_{2}\right]}$

Also we have $\Delta A_{t}^{H}=A_{t+1}^{H}-A_{t}^{H}=\frac{\gamma \pi_{1}^{t}\left(\phi_{1}^{t+1}-\phi_{1}^{t}\right)}{\mu\left[p \pi_{1}^{t}+(1-p) \pi_{0}^{t}\right]+\gamma}=\frac{\gamma \pi_{1}^{t} \lambda \phi_{0}^{t}}{\mu\left[(2 p-1) \pi_{1}^{t}+(1-p)\right]+\gamma}=\frac{\gamma \pi_{1}^{t} \lambda \phi_{0}^{t}}{K_{1} \pi_{1}^{t}+K_{2}}$

Hence $\Delta A_{t}^{T}>\Delta A_{t}^{H} \Leftrightarrow \frac{\left(\mu p+\gamma \phi_{1}^{t}\right) K_{2} \delta \pi_{1}^{t}+\gamma \lambda \phi_{0}^{t}(1+\delta) \pi_{1}^{t}\left(K_{1} \pi_{1}^{t}+K_{2}\right)}{\left[K_{1}(1+\delta) \pi_{1}^{t}+K_{2}\right]\left[K_{1} \pi_{1}^{t}+K_{2}\right]}>\frac{\gamma \pi_{1}^{t} \lambda \phi_{0}^{t}}{K_{1} \pi_{1}^{t}+K_{2}}$ $\Leftrightarrow \frac{\left(\mu p+\gamma \phi_{1}^{t}\right) K_{2} \delta \pi_{1}^{t}+\gamma \lambda \phi_{0}^{t}(1+\delta) \pi_{1}^{t}\left(K_{1} \pi_{1}^{t}+K_{2}\right)}{\left[K_{1}(1+\delta) \pi_{1}^{t}+K_{2}\right]\left[K_{1} \pi_{1}^{t}+K_{2}\right]}>\frac{\gamma \pi_{1}^{t} \lambda \phi_{0}^{t}\left[K_{1}(1+\delta) \pi_{1}^{t}+K_{2}\right]}{\left[K_{1} \pi_{1}^{t}+K_{2}\right]\left[K_{1}\left(\pi_{1}^{t}+\lambda \pi_{0}^{t}\right)+K_{2}\right]}$ $\Leftarrow(1+\delta) \pi_{1}^{t}\left(K_{1} \pi_{1}^{t}+K_{2}\right)>\pi_{1}^{t}\left[K_{1}(1+\delta) \pi_{1}^{t}+K_{2}\right] \Leftrightarrow(1+\delta) K_{1} \pi_{1}^{t}+(1+\delta) K_{2}>K_{1}(1+\delta) \pi_{1}^{t}+K_{2} \Leftrightarrow \delta K_{2}>0 \quad$, which is obviously true.

$\Delta B_{t}^{T}=\frac{\gamma}{\mu+\gamma}\left[\left(\phi_{1}^{t}+\lambda \phi_{0}^{t}\right)(1+\delta) \pi_{1}^{t}-\phi_{1}^{t} \pi_{1}^{t}\right], \Delta B_{t}^{H}=\frac{\gamma}{\mu+\gamma}\left[\left(\phi_{1}^{t}+\lambda \phi_{0}^{t}\right) \pi_{1}^{t}-\phi_{1}^{t} \pi_{1}^{t}\right]$. Therefore $\Delta B_{t}^{T}>\Delta B_{t}^{H}$ can be derived from the obvious fact $1+\delta>1$.

There is no monotonous result about the inequality between $\Delta B_{t}^{T}$ and $\Delta B_{t}^{N}$. 
(ii) Suppose at time $t$ a sell order comes to the market.

In the normal phase we have $\phi_{1, N}^{t+1}=\frac{\mu \phi_{1}^{t}\left(p \pi_{0}^{t}+(1-p) \pi_{1}^{t}\right)+\gamma \phi_{1}^{t}}{\mu\left[\phi_{0}^{t}+\phi_{1}^{t}\left(p \pi_{0}^{t}+(1-p) \pi_{1}^{t}\right)\right]+\gamma}=\left(1-\rho_{1}\right) \phi_{1}^{t} \quad$ (with $\rho_{1}=\frac{\mu \phi_{0}^{t}\left[(1-p) \pi_{0}^{t}+p \pi_{1}^{t}\right]+\gamma}{\mu\left[\phi_{0}^{t}+\phi_{1}^{t}\left(p \pi_{0}^{t}+(1-p) \pi_{1}^{t}\right)\right]+\gamma} \quad$ and $\pi_{1, N}^{t+1}=\frac{\mu \pi_{1}^{t}(1-p)+\gamma \pi_{1}^{t}}{\mu\left[p \pi_{0}^{t}+(1-p) \pi_{1}^{t}\right]+\gamma}=(1-\delta) \pi_{1}^{t} \quad$ (with
$\left.\delta=\frac{\mu \pi_{0}^{t}(2 p-1)}{\mu\left[p \pi_{0}^{t}+(1-p) \pi_{1}^{t}\right]+\gamma}>0\right)$. During the transitional phase and herd phase we have $\phi_{1, T}^{t+1}=\phi_{1, H}^{t+1}=\frac{\gamma}{\mu+\gamma} \phi_{1}^{t}$ $=\left(1-\rho_{2}\right) \phi_{1}^{t}$, with $\left.\rho_{2}=\frac{\mu}{\mu+\gamma}>\rho_{1}>0\right)$ and $\pi_{1, T}^{t+1}=\pi_{1, H}^{t+1}=\pi_{1}^{t}$, since during the herd and transition phase the informed act upon only because they receive the clear signal $w=1$, regardless of their noisy signal $v$.

Please remember now $\left|\Delta A_{t}\right|=A_{t}-A_{t+1}$ and $\left|\Delta B_{t}\right|=B_{t}-B_{t+1}$ since the market maker will decrease the price when the just-presented order is sell. Hence we have $\left|\Delta A_{t}^{T}\right|=\left|\Delta A_{t}^{H}\right|=\frac{\gamma \rho_{2} \phi_{1}^{t} \pi_{1}^{t}}{\mu\left[p \pi_{1}^{t}+(1-p) \pi_{0}^{t}\right]+\gamma}$. When we compare $\left|\Delta A_{t}^{T}\right|$ and $\left|\Delta A_{t}^{N}\right|$, we should notice that if we hold everything else equal, we have $A_{t}^{T}=A_{t}^{N}$. So we need only to compare $A_{t+1}^{T}$ and $A_{t+1}^{N}$ in order to tell the inequality of $\left|\Delta A_{t}^{T}\right|$ and $\left|\Delta A_{t}^{N}\right|$. In the transition phase the sell can only come from the informed with signal $w=0$, the very bad news, while in the normal phase the sell is from the very bad news or the bad news. So it is obviously that $A_{t+1}^{T}<A_{t+1}^{N}$, which means $\left|\Delta A_{t}^{T}\right|$ $>\left|\Delta A_{t}^{N}\right|$. We also have $\left|\Delta B_{t}^{T}\right|=\left|\Delta B_{t}^{H}\right|$ because the pricing formula and the update of $\phi_{1}^{t}$ and $\pi_{1}^{t}$ are exactly the same. Again, there is no monotonous result about the inequality between $\Delta B_{t}^{T}$ and $\Delta B_{t}^{N}$.

\section{Proof of Proposition 6}

It is straightforward to understand that during the normal and herd phase the arrival of the agents who decide not to trade will not cause any change in the price quote, since these agents can only be uninformed traders who bring no new information about the asset payoff. In the transition phase, however, the informed trader with signal $w=1 \& v=0$ will not trade, while they do bring new information about the asset payoff to the market maker. Hence the price will change correspondingly. After the arrival of a no-trading agent in the transition phase, the market maker will adjust his assessed distribution on $W$ and $V$ as

$\phi_{1}^{t+1}=\frac{\mu\left[(1-p) \pi_{1}^{t}+p \pi_{0}^{t}\right]+\gamma \phi_{1}^{t}}{\mu\left[(1-p) \pi_{1}^{t}+p \pi_{0}^{t}\right]+\gamma}$ and $\pi_{1}^{t+1}=\frac{\mu \pi_{1}^{t}(1-p)+\gamma \pi_{1}^{t}}{\mu\left[(1-p) \pi_{1}^{t}+p \pi_{0}^{t}\right]+\gamma}$. In order to simplify the calculation, we define $\beta_{0}^{t}=p \pi_{0}^{t}+(1-p) \pi_{1}^{t}$ and $\beta_{1}^{t}=(1-p) \pi_{0}^{t}+p \pi_{1}^{t}$. It is easy to conclude that $1-p<\beta_{0}^{t}, \beta_{1}^{t}<p$ Then we rewrite $\phi_{1}^{t+1}=\frac{\mu \beta_{0}^{t}+\gamma \phi_{1}^{t}}{\mu \beta_{0}^{t}+\gamma}=\phi_{1}^{t}+g \phi_{0}^{t} \quad\left(\right.$ with $g=\frac{\mu \beta_{0}^{t}}{\mu \beta_{0}^{t}+\gamma}>0$ ) and $\pi_{1}^{t+1}=\frac{\mu(1-p)+\gamma}{\mu \beta_{0}^{t}+\gamma} \pi_{1}^{t}=(1-\delta) \pi_{1}^{t} \quad\left(\right.$ with $\left.\delta=\frac{\mu \pi_{0}^{t}(2 p-1)}{\mu \beta_{0}^{t}+\gamma}>0\right)$.

Hence $B_{t+1}^{T}=\frac{\gamma \phi_{1}^{t+1} \pi_{1}^{t+1}}{\mu+\gamma}=\frac{\gamma}{\mu+\gamma}\left(\phi_{1}^{t}+g \phi_{0}^{t}\right)(1-\delta) \pi_{1}^{t}=\frac{\gamma \phi_{1}^{t} \pi_{1}^{t}}{\mu+\gamma}\left(1+g \phi_{0}^{t} / \phi_{1}^{t}\right)(1-\delta)=\left(1+g \phi_{0}^{t} / \phi_{1}^{t}\right)(1-\delta) B_{t}^{T}$ $\therefore B_{t+1}^{T}>B_{t}^{T} \Leftrightarrow\left(1+g \frac{\phi_{0}^{t}}{\phi_{1}^{t}}\right)(1-\delta)>1 \Leftrightarrow 1+g \frac{\phi_{0}^{t}}{\phi_{1}^{t}}>\frac{1}{1-\delta} \quad \Leftrightarrow \frac{\phi_{0}^{t}}{\phi_{1}^{t}}>\frac{1}{g}\left(\frac{1}{1-\delta}-1\right) \quad \Leftrightarrow \frac{1-\phi_{1}^{t}}{\phi_{1}^{t}}>\frac{1}{g(1-\delta)}-\frac{1}{g}=\frac{\delta}{g(1-\delta)}$ $\Leftrightarrow g(1-\delta)-g(1-\delta) \phi_{1}^{t}>\delta \phi_{1}^{t} \Leftrightarrow(\delta+g-\delta g) \phi_{1}^{t}<g(1-\delta) \Leftrightarrow \phi_{1}^{t}<\frac{g(1-\delta)}{\delta+g-\delta g}$ and $B_{t+1}^{T}<B_{t}^{T} \Leftrightarrow \phi_{1}^{t}>\frac{g(1-\delta)}{\delta+g-\delta g}$

Also $A_{t+1}^{T}=\frac{\mu p \pi_{1}^{t+1}+\gamma \phi_{1}^{t+1} \pi_{1}^{t+1}}{\mu\left[p \pi_{1}^{t+1}+(1-p) \pi_{0}^{t+1}\right]+\gamma}=\frac{\mu p+\gamma \phi_{1}^{t+1}}{\mu \beta_{1}^{t+1}+\gamma} \pi_{1}^{t+1}=\frac{\mu p+\gamma\left(\phi_{1}^{t}+g \phi_{0}^{t}\right)}{\mu\left[\beta_{1}^{t}-(2 p-1) \delta \pi_{1}^{t}\right]+\gamma}(1-\delta) \pi_{1}^{t}$

$\therefore A_{t+1}^{T}>A_{t}^{T} \Leftrightarrow \frac{\mu p+\gamma\left(\phi_{1}^{t}+g \phi_{0}^{t}\right)}{\mu\left[\beta_{1}^{t}-(2 p-1) \delta \pi_{1}^{t}\right]+\gamma}(1-\delta) \pi_{1}^{t}>\frac{\mu p+\gamma \phi_{1}^{t}}{\mu \beta_{1}^{t}+\gamma} \pi_{1}^{t}$

$\Leftrightarrow \mu p+\gamma\left(\phi_{1}^{t}+g \phi_{0}^{t}\right)>\frac{\mu\left[\beta_{1}^{t}-(2 p-1) \delta \pi_{1}^{t}\right]+\gamma}{(1-\delta)\left(\mu \beta_{1}^{t}+\gamma\right)}\left(\mu p+\gamma \phi_{1}^{t}\right)$

$\Leftrightarrow \mu p+\gamma\left[(1-g) \phi_{1}^{t}+g\right]>h\left(\mu p+\gamma \phi_{1}^{t}\right) \quad\left(\right.$ define $\left.\quad h=\frac{\mu\left[\beta_{1}^{t}-(2 p-1) \delta \pi_{1}^{t}\right]+\gamma}{(1-\delta)\left(\mu \beta_{1}^{t}+\gamma\right)}\right)$ 
$\Leftrightarrow \gamma[h-(1-g)] \phi_{1}^{t}<\mu p(1-h)+\gamma g \Leftrightarrow \phi_{1}^{t}<\frac{\mu p(1-h)+\gamma g}{\gamma(h+g-1)}$

and $A_{t+1}^{T}<A_{t}^{T} \Leftrightarrow \phi_{1}^{t}>\frac{\mu p(1-h)+\gamma g}{\gamma(h+g-1)}$ 